\title{
Classification and decision making of medical infrared thermal images
}

\author{
Ricardo Vardasca ${ }^{1,2}$, Lucia $\operatorname{Vaz}^{3}$, Joaquim Mendes ${ }^{4}$ \\ ${ }^{1}$ LABIOMEP, UISPA-LAETA-INEGI, Faculdade de Engenharia, Universidade do Porto, \\ Rua Dr. Roberto Frias S/N, 4200-465 Porto, Portugal \\ ${ }^{2}$ Medical Imaging Research Unit, Faculty of Computing, University of South Wales, \\ Trefforest Campus, Pontypridd, Rhondda Cynon Taff, CF37 1DL, Wales, United Kingdom \\ rvardasca@fe.up.pt \\ ${ }^{3}$ Faculdade de Engenharia, Universidade do Porto, Rua Dr. Roberto Frias S/N, 4200-465 \\ Porto, Portugal \\ ee11155@fe.up.pt \\ ${ }^{4}$ LABIOMEP, UISPA-LAETA-INEGI, Faculdade de Engenharia, Universidade do Porto, \\ Rua Dr. Roberto Frias S/N, 4200-465 Porto, Portugal \\ jgabriel@fe.up.pt
}

\begin{abstract}
Medical Infrared Thermal Imaging (MITI) is a technique that allows to record skin surface temperature distribution, in a completely safe and innocuous manner. These images provide underlining physiological in-formation on the blood flow, vasoconstriction/vasodilatation, inflammation, transpiration or other processes that can contribute to the skin temperature. This medical imaging modality has been available for nearly six decades and proved to be useful for vascular, neurological and musculoskeletal conditions. Since the recordings are digital, in a form of a matrix of numbers (image), it can be computationally analyzed, by a specialist mostly performing operations of processing and analysis manually supported by proprietary solutions of software. This limits the number of images that can be processed, making difficult the evolution of knowledge, expertise development and information sharing. This chapter aims to disclose the medical imaging method, along with its particularities, principles, applications, advantages and disadvantages. Introducing all available classification and decision making methods that can be employed in digital information along with a literature review of their operation in the biomedical applications of Infrared Thermal Imaging.
\end{abstract}

Keywords: Biomedical applications, Classifiers, Data-mining, Infrared thermal imaging, Medical thermography 


\section{Introduction}

Thermography is an imaging technique that can record remotely the temperature distribution of a surface. In the scope of this publication, it is applied to the human body and its largest organ, the skin, which makes the interface between the inners structures and the surrounding environment.

It is aim of this work to briefly introduce the history of MITI, the physical principles of infrared thermography, the operational aspects of its application in medicine, the different data-mining classifiers. The considered for this overview were: Artificial Neural Network (ANN), Support Vector Machine (SVM), k-Nearest Neighbor (k-NN), Naïve Bayes (NB) and Adaboost, along with their usage to label MITI data found on PUBMED and SCOPUS literature sources, the chapter is finalized with a final discussion about the strengths, weaknesses and opportunities of applications of intelligent data classifiers to MITI data.

\subsection{Classification in Bio-Apps}

The MITI applications have been growing since its first introduction in 1956, with the development of classification methods to classify data, which reduce human error and are able to deal with large amounts of data, this work intends to first provide an overview of the MITI technique and its applications that have used data classifiers to improve the outcomes. Future challenges in this research area are outlined.

\subsection{Historical perspective}

The Skin temperature can be intended as a physiological aspect and indicator of human body that is related with healthy or pathological state. Hippocrates is known for being the father of conventional medicine. He also performed what can be called the first registry of skin temperature using mud baths, the warmer regions would dry firstly than the cold, and stated the sentence "In whatever part of the human body, excess of heat or cold is felt, the disease is there to be discovered" (Qi and Diakides 2007).

In 1800 Sir William Herschel, a German born British astronomer, who attracted by the Sir Isaac Newton experiment, in exposing white light through a glass prism resulting in a colored rainbow, decided to measure the temperatures of those rainbow colors with thermometers, having found that the color presenting the highest temperature value was the red and beyond this color there was an invisible light with a highest temperature value, being this way discovered the infrared radiation. Sir John Herschel, son of William, in 1840 performed the first image of thermal radiation when focusing solar radiation with a lens into a suspension of carbon 
particles in alcohol, this technique was called evaporography and the resulting image, a thermogram (Ring 2007).

The bolometer, an instrument that is able to determine the power of incident electromagnetic radiation through the heating of a material with a temperaturedependent electrical resistance, was invented in 1880 by Samuel Langley. This device become a very sensitive thermometer, its discoverer claimed that it could estimate the heat irradiated by a cow that is at 400 meters' distance (Richards 1994). In the development of thermal cameras this sensor was very relevant.

A Hungarian physicist, Kálmán Tihanyi, in 1929 invented in Britain the first infrared camera as a night vision instrument to be used for anti-aircraft defense (Lisowska-Lis et al. 2011). A few years later, the evaporograph (can be intended as the first conventional IR camera), was developed for military use only by Marianus Czerny (German physicist). The first thermograph device using a 16-inch searchlight reflector, a dual axis scanner, and a bolometer detector was built by the US Army in 1952 (Lloyd 2013).

The first known application of usage of a thermographic system in medicine examining the human body skin temperature was performed by Ray Lawson (Canadian physician) in 1956, when he used an evaporograph to assess the temperature distribution of a breast cancer (BC) malignant tumor (Lawson 1956).

In the proceedings of the first conference in New York about Thermography and its clinical applications in 1963, Lloyd Williams, a British physician, proposed as research areas of interest and application for medical thermography: breast disease, burns, peripheral vascular disease, varicose veins, vasomotor control, thyrotoxicosis and rheumatoid arthritis (Williams 1964).

Medical Infrared Thermal Imaging (MITI) is a medical imaging modality that records large areas of surface skin temperature not involving any contact or induced radiation, being a fast exam and innocuous to the patient. It allows to monitor physiology in real-time providing a window for the autonomic nervous system (Jones and Plassmann 2002, Ring and Ammer 2000, Ring and Ammer 2012).

\section{$2 \quad$ Infrared imaging}

\subsection{Physical principles}

This imaging technique relies in the principles of thermal physics, which are now described.

Maxwell demonstrated the existence of electromagnetic waves in free space and media. These waves in free space can be classified by their frequency and wavelength and related with the free space propagation of the velocity of light. The electromagnetic radiation frequency is given by the equation 1 : 


$$
f=\frac{E}{h}
$$

, where $\mathrm{f}$ is the frequency, $\mathrm{E}$ is the energy and $\mathrm{h}$ is the Planck's constant $(6.6 \mathrm{x}$ $\left.10^{-34} \mathrm{~J} . \mathrm{s}\right)$.

The frequency obtained by the equation (1) of emission or absorption of radiation is generated by transitions from one energy level to another (Thomas 1999).

The electromagnetic spectrum is a representation of the characteristic distribution of electromagnetic radiation emitted or absorbed by a particular object in terms of frequency, wavelength and photon energy. Fig. 1, on the top, represents the regions of the spectrum, on the left with higher frequency, lower wavelength and lower photon energy are the Gamma rays; and on the opposite side with lower frequency, higher wavelength and higher photon energy are the Radio waves. The human eyes can only perceive the visible region of the electromagnetic spectrum (wavelength from 0.7 to $0.4 \mu \mathrm{m}$ ), the Infrared spectrum is located immediately at the right of the visible region and can be divided in three sub-regions (fig. 1 bottom): Near Infrared (wavelength from 0.7 to $3 \mu \mathrm{m}$ ), Mid Infrared (wavelength from 3 to $8 \mu \mathrm{m}$ ) and Long Infra-red (wavelength from 0.7 to $3 \mu \mathrm{m}$ ).

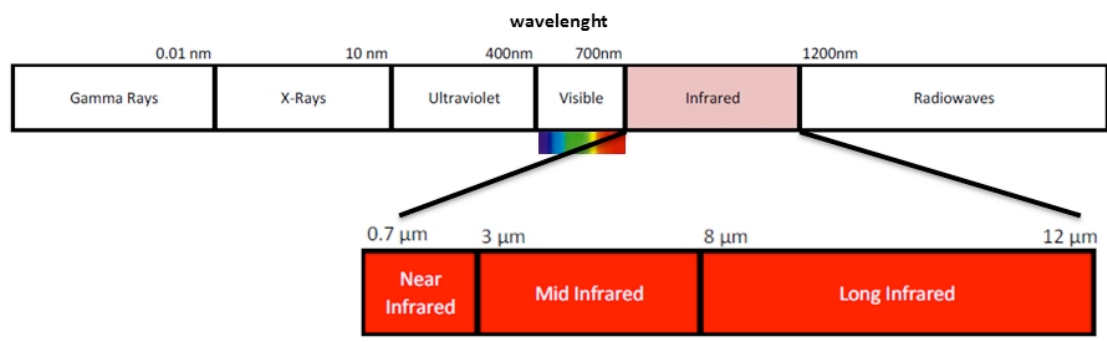

Fig. 1 Electromagnetic spectrum with emphasis in the infrared, distributed by wavelength.

Any object at a temperature above absolute zero has the ability of emitting electromagnetic radiation. This works through converting the object's thermal energy into electromagnetic energy, or thermal radiation. Through the electromagnetic spectrum, all objects in it have the ability to emit and absorb electromagnetic radiation. A blackbody is an ideal object that absorbs all incident electromagnetic radiation, at all wavelengths, and at a uniform temperature, being the best possible thermal radiator, having in consideration the Kirchhoff's Law, which establishes an equity between the amount of absorption and the amount of emission.

All matter absorbs electromagnetic radiation until a certain degree. A blackbody emission, also called blackbody radiation, has the particular characteristic of the frequency distribution depending only on the temperature. This blackbody ra- 
diation can be described though three physical laws: Planck's radiation law, Wien's displacement law and Stefan-Boltzmann formula. The amount of energy emitted by a black body in radiation of a certain wavelength (equation 2) or frequency (equation 3) is given by the Planck's law, which has two forms as represented in table 1.

Table 1. The Planck Law represented by formula and graph as function of electromagnetic radiation wave-length and frequency. Being "I" the energy per unit time (or the power) radiated per unit area of emitting surface in the normal direction per unit solid angle per unit (" $\lambda$ " - wavelength, "v" - frequency) by a black body at temperature " $T$ ". "h" is the Planck constant, "c" is the speed of light in a vacuum $(299792458 \mathrm{~m} / \mathrm{s})$ and " $k$ " is the Boltzmann constant $(1.3806488(13) \times 10-23 \mathrm{~J} \quad \mathrm{~K}-1)$.

As a function of wavelength

(b)

As a function of frequency

$$
I(\lambda, T)=\frac{2 h c^{2}}{\lambda^{5}}
$$

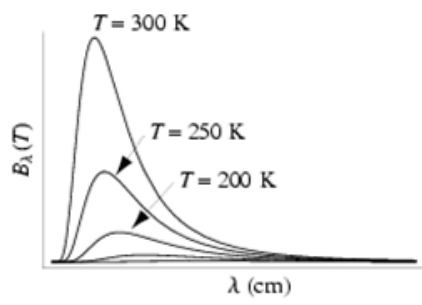

$$
I(v, T)=\frac{2 h v^{3}}{c^{2}} \frac{1}{e^{\frac{h v}{\lambda k T}}-1}
$$

The Planck's law provides the amount of radiated energy by a blackbody at all frequencies, their intensity rapidly bear to zero for high and low frequencies or short and long wavelengths. This means that at room temperature, a blackbody does not emit any radiation in the visible spectrum range.

The Wien's displacement law is represented by the equation 4:

$$
\lambda_{\max }=\frac{\mathrm{b}}{T}
$$

, where $\lambda$ max corresponds to the wavelength were maximum intensity of the radiation produced by a blackbody occurs, $\mathrm{b}$ is the Wien's displacement constant $\left(2.8977721(26) \times 10^{-3} \mathrm{~m} \mathrm{.K}\right)$ and $\mathrm{T}$ is the known temperature.

The fig. 2 represents the maximum amount of radiation $(\lambda \max )$ per wavelength calculated by Wien's displacement law based on its temperature.

The Stefan-Boltzmann equation (5) is: 


$$
E=\varepsilon \sigma T^{4}
$$

, where $\mathrm{E}$ is the total radiated energy from a blackbody [W $\left.\mathrm{m}^{2}\right], \varepsilon$ is the emissivity value, $\sigma$ is the Stefan-Boltzmann constant $\left[5.6696 \times 10^{-8} \mathrm{~W} \mathrm{~m}^{-2} \mathrm{~K}^{-4}\right]$ and $\mathrm{T}$ the absolute temperature. The $\mathrm{E}$ represents the total radiated energy from a blackbody, per unit area and time, being proportional to the fourth power of its absolute temperature. The vast majority of the existing radiation thermometers are based in this equation.

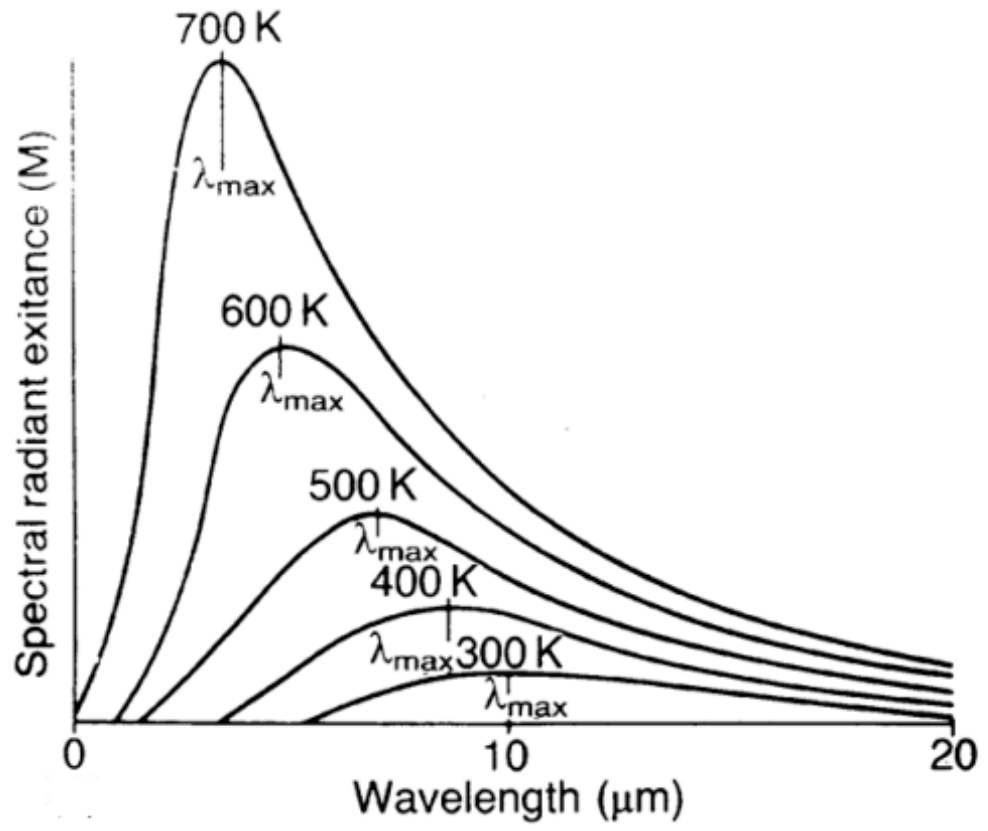

Fig. 2 Wien's displacement law representing the maximum amount of radiation per wavelength.

Real objects differ from the blackbody formulae over an extended wave-length region, despite that in certain spectral intervals they can approach a blackbody behavior. There are three important factors, represented by fig. 3 , to take in consideration (Thomas 1999):

Spectral absorption $(\alpha)$ - where a portion of the incident radiation on an object can be absorbed, originating as a result the ratio between the spectral radiant power absorbed by an object to the incident radiation on it.

Spectral reflectance $(\varrho)$ - where a portion of the incident radiation on an object can be reflected, originating as a result the ratio between the spectral radiant power reflected by an object to the incident radiation on it. 
Spectral transmittance $(\tau)$ - where a portion of the incident radiation on an object can be transmitted, originating as a result the ratio between the spectral radiant power transmitted through an object to that radiation incident on it.

The relationship between these processes is of such importance that they should always be added up to the whole at any wavelength (Kirchhoff's law) in order to correct the measured/calculated temperature.

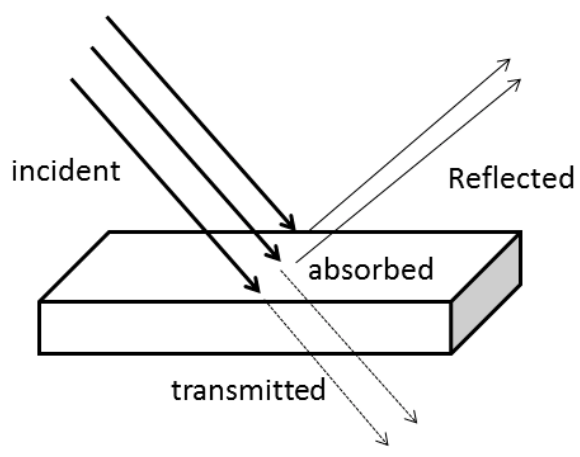

Fig. 3 The alternative pathways of radiation falling in an object surface.

By the Kirchhoff's law equation (6):

$\alpha+\varrho+\tau=1$

, which establishes an equilibrium between the processes, for opaque materials, where $\tau=0$, the equation can be simplified to (7):

$$
\alpha+\varrho=1
$$

When estimating the temperature of an object returning contact, the knowledge of the emissivity value of this object is of extreme importance. The emissivity of an object is the ratio of the radiant emission produced by an object by that of a black body at a specific temperature, its value varies between 0 and 1 and is influenced by the color, shape and surface of the object. This parameter considerably affects the accuracy of the measurement. A perfect black body has 1 as emissivity value, being considered a perfect emitter. For highly reflective objects, the emissivity value tends to zero. A surface that reflects infrared energy has an emissivity value of less than 1 , constant with wave-length, is considered a grey body, and its total radiated energy can be obtained adjusting the object emissivity in StefanBoltzmann, equation 5 (Thomas 1999).

Infrared camera image file formats are all proprietary and besides storing the radiometric reading per each array sensor element, they store other data such as the equipment name, serial number, date, time, focal length, emissivity, object dis- 
tance, reflected apparent temperature, atmospheric temperature, relative humidity, camera temperature range, field of view, focus distance, 5 Planck constants (R1, $\mathrm{B}, \mathrm{F}, \mathrm{R} 2$ and $\mathrm{O}$ ) and matrix of radiometric values that corresponds to the sensor array size.

Per each infrared sensor, a voltage is perceived from the thermal energy detected and quantified in a 16-bit number, which will be the radiometric measurement value.

Based in these values, and for obtaining the temperature, the raw reflection temperature (Reflt) has be calculated according with the equation 8 :

$$
R e f l t=\frac{R 1}{R 2^{*} e^{\frac{B}{\text { Temperature reflected }}}-F}-O
$$

Along with this value, since the emissivity value for the human skin is $<1$, being worldwide accepted the value of 0.98 , it is needed to calculate the correct object raw radiometric value $(\mathrm{S})$, with the equation 9:

$$
S=\frac{(\text { radiometric raw }-(1-\text { Emissivity }) * r e f l t)}{\text { Emissivity }}
$$

With the raw reflection temperature and the object raw radiometric value, the pixel value, which corresponds to a single sensor, the estimated temperature can be obtained with the equation 10 :

$$
\text { Temperature }(K)=\frac{B}{\ln \left(\frac{R 1}{S+O}+1\right)}
$$

This temperature can be translated from SI unit to the most common unit, Celsius $\left({ }^{\circ} \mathrm{C}\right)$, by the equation 11 :

Temperature $\left({ }^{\circ} \mathrm{C}\right)=273.15$-Temperature $(K)$

\subsection{Operational aspects in medicine}

The imaging concept of generating temperature in infrared images was just described before, for a valid medical thermal examination, a strict image capture protocol has to be followed (Ring and Ammer 2012). It consists of preparation of the examination room, preparation of the capture equipment, preparation of the subject before and during the accomplishment of the collection of the images and the way of capturing and storing them. 
In the existing literature, there is a gap of reference values in the distribution of normal and pathological values of skin temperature in humans. Infrared thermal imaging, unlike any other medical imaging modality such as digital radiology, does not yet have a set of globally accepted standard positions to improve the quality of assessments and increase its usage. Although it is obvious that the standardization of the technique would reduce substantially the errors. Literature describe the reproducibility of the technique only vaguely (Ammer 2003).

There are environmental conditions that can affect the examination outcomes, those are: room temperature, relative humidity and air circulation flow, as well as the examination room intrinsic conditions (e.g. false ceilings where air may be filtered and no direct airflow is projected towards the subject, double glazed windows and illumination).

Patient preparation and clothing have an influence in the measurements, which can be addressed through pre-examination acclimatization period. The imaging system has to comply to quality assurance requirements to avoid affecting the process of acquiring the image (Ring et al. 2007). The use of regions of interest (ROI) masks strengthens the quality of data collection, also facilitating the correct positioning and distance between equipment and the subject being examined, as well as the adjustment to the size and position of the ROI. All these aspects must be taken into consideration in order to homogenize the image capture and analysis process in order to make comparisons possible and allow the generation of reference data (Ring et al. 2004), otherwise it has to be achieved by image processing techniques that have an associated error.

In the UK, a project had a goal of building a reference images database of the distribution of skin temperature in ROIs of human body (Ring et al. 2005), 24 views of the human body were defined with the corresponding ROIs (Ammer 2008) and the following aspects of medical thermal imaging were standardized.

According to Ring et al. (Ring et al. 2004) the usage of standard procedures reduces the amount and influence of variables, promotes understanding and knowledge exchange, and enforces repeatability. In medical thermal imaging this is a relevant aspect, since most of past errors were made due to the absence of a standard image capturing methodology (Ammer 2003). This had led to a decreased application of this imaging method in medicine and a considerable decrease in credibility among health professionals.

In 2006, the American Academy of Thermology (AAT) suggested a document (Schwartz et al. 2006) which specifies the purpose, common indications, contraindications and limitations of using Infrared Thermography in medicine.

The examination room should have a stable temperature and relative humidity system, which should maintain in values of $22 \pm 1^{\circ} \mathrm{C}$ and below $50 \%$ respectively, the air flow should be inferior to $2 \mathrm{~m} / \mathrm{s}$ and the illumination should have protections to avoid incident lightning over the subject. The outside laboratory windows have to be completely shut, to prevent solar radiation, but also enforce privacy during the procedure. The provision of a disrobing room at same environmental conditions of the examination main room is advised for the subject rest during the 
acclimatization period, which is advised to be of 15 minutes before the examination to facilitate thermal steadiness. In order to ensure the adequate space for the procedure of collecting the images the whole unnecessary equipment must be removed from the room area to prevent unwanted heat reflections. The subject should be away from equipment's and walls, as far as possible, to decrease the thermal reflections. The minimum necessary furniture is recommended to provide adequate space for maneuver of the equipment and allow sufficient distance between the camera and the subject. For avoiding external disturbances during the recording procedure, a signaling at the door with the "Examination in progress" is recommended.

Before the appointment, the subject requires some care, such as avoid smoking, drinking hot drinks (coffee and tea) or having an heavy meal two hours (minimum) prior to the investigation, since this would influence the metabolism; avoid any moistures on the skin, since these substances will thermally insulate the skin; avoid alcohol or drugs consumption and physiotherapy or sports on the images collection day, once this would affect the subject thermoregulation; and report any infections and any medicines taken, independently of the source of their prescription, considering that they might be vasodilators or vasoconstrictors.

On arrival, the subject should remove the clothing according with the body view of interest and he should be comfortable with (changing division is recommended) and in line with the area of the part of the body to be screened. It should be avoided to have jewelry, crossing legs, or hands/arms close or on the body and touching the body. Tight clothing or seating should be abstained to prevent marks that can result in skin temperature changes.

All recorded data has to be correctly stored and the subject privacy and confidentiality must be respected and assured. The images are then analyzed using ROIs were parameters such as mean, minimal, median, maximum temperature, standard deviation, kurtosis, skewness and histogram are calculated.

\subsection{Image capture devices}

There are two main types of infrared thermal cameras, which are the called "cooled systems", which the sensors operate at a lower temperature than the environment, produce images of higher quality, have better sensitivity but have a limited time of operation due to the refrigeration system and are costly when compared with the "uncooled systems", that have no limitations in usage and normally present sensor focal plane arrays of higher dimensions, their images quality over time become closer to the "cooled" equipment.

Before the image collection procedure, the investigator has to verify if all needed equipment is set up adequately (the usage of a check list is recommended). The infrared camera has to switched on at least 15/20 minutes before the first image to prevent start-up drift effect and allow electronic equilibrium of the camera components; this can be tested for the used camera according to the quality assurance 
guidelines (Ring et al. 2007). An image of the calibration source is recommended before starting the recording process of the subject, it will also apply to the end of the subject imaging, when another image should be taken from the blackbody under the same condition of the first, comparing both will allow to check for recording errors.

In order to capture the desired views, a correct distance and angle from the camera to the target, its position and field of view of the recording equipment has to be obtained. This arrangement procedure can be somewhat simplified through the usage of software capture overlay masks over the live displaying. Moreover, the usage of camera stand will facilitate quick and easy positioning and stable fixation onto the target view, avoiding angles change.

The infrared camera minimal requirements for medical use have been established (Howell and Smith 2009, TC121 et al. 2008, ISO and TR13154 2009) and indicate a minimum sensor array size of $320 \times 240$, NETD of $<50 \mathrm{mK}$ at $30^{\circ} \mathrm{C}$ and repeatability of $\pm 2 \%$ of the overall reading. The lenses used in these imaging systems are standard lens (IR-lens $24^{\circ}$ ) for body partial views and wide angle lens (IR-lens $45^{\circ}$ ) for full body views.

It is also important to have a hygrometer with a large display to verify the environmental condition of the examination room.

\section{Classification Methods on MITI}

Advanced data analysis techniques provide a diversity of distinct approaches for data reasoning and selection. Those are being used increasingly in problem domains involving classification and within a set of ostensibly unrelated data can find shared features.

All of the different methods are based in input variables, which might or not been reduced, that are subject to operators that divide that variables into groups, providing a classification to each.

In MITI the common input variables are taken from ROIs in the images, which can be first order statistics such as mean (average) temperature, standard deviation, entropy, kurtosis, skewness and variance, or second order statistics such as homogeneity, contrast, energy, correlation, entropy and maximum probability.

In order to reduce or simplify the variables, statistical transformations can be used such as Principal Component Analysis (PCA), Nonparametric Discriminant Analysis (NDA), Linear Discriminant Analysis (LDA) or Information Gain Ratio (IGR).

The accomplishment of the classifiers is measured in premises of accuracy, sensitivity and specificity, which are calculated from the amount of true and false positives, true and false negatives and receiver operating characteristic (ROC). 
The first known publication ( $\mathrm{Ng}$ et al. 2002) which related classifiers with MITI, dated on 2002, presented the usage of an ANN with the purpose of identifying $\mathrm{BC}$ in thermal images.

\subsection{ANN}

The largest artificial intelligence method for data classification in MITI is based on ANN. An ANN can be intended as a computational approximation (model) of the human brain (Hassoun 1995). In similarly with a brain, an ANN is composed of neurons and inter-connections. An illustrative format of such format is composed of neurons, represented as nodes, and interconnections, represented by edges (Fig. 4). Several variations of ANNs exist, being distinct in their topology, orientation of connections and approaches for learning. The prominent is the 'feed-forward back-propagation', which is also called multilayer perceptron. Because of its efficiency and somewhat uncomplicated training process.

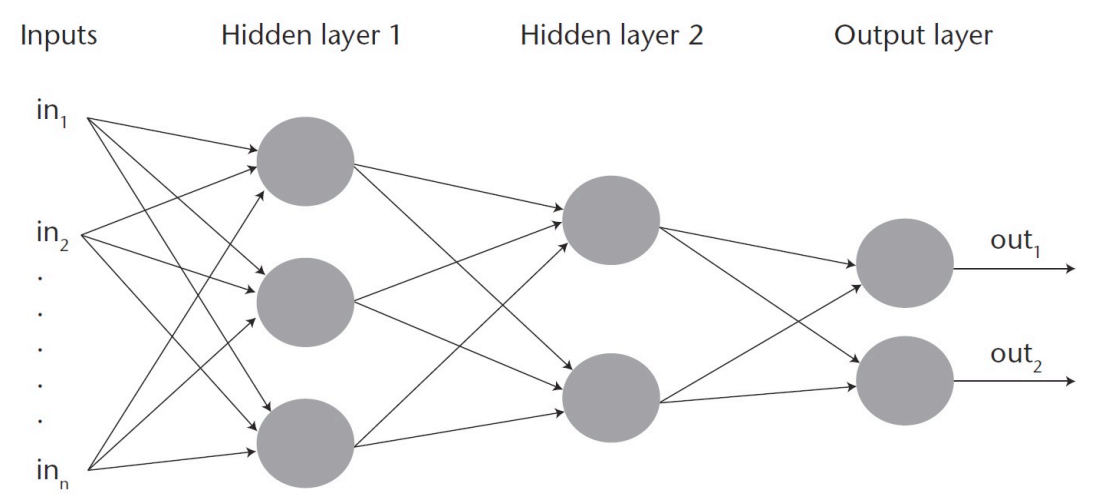

Fig. 4 A representation of an artificial neural network (multiplayer perception).

A description in detail of a multilayer perceptron ANN is besides the ambit of this chapter and is well detailed in related existing literature. For an improved understanding, an ANN can be adaptively trained for its parameters by machine learning procedures; hence, consequently, it can learn to assertively classify input elements, usually in numerical format (Hassoun 1995). To assess the degree of confidence of an ANN, the data set to be classified should generally be divided into two groups. The first should consist of cases used for the training process, by which ANN will adapt. While the second grouping is composed of cases that are totally unknown to the ANN, which has undergone a learning process. These data are subject to classification and their results are compared with the actual values of the class to which they belong. Theoretically, if the training set is large enough, an ANN can cluster enough knowledge to classify elements that are totally unknown (Hassoun 1995). 
In specific, the infrared heat radiation and physiologic data are the basic elements for the training of the ANNs by processing backpropagation algorithms. Each ANN created had three layers (input, hidden and output) and the processing elements were propagated in the network by output layer until input layer, without interconnection between processing elements in the same layer ( $\mathrm{Ng}$ et al. 2002).

In detail, the basic variables used were obtained by the correlation between the captured basic elements, i.e. diagnostic outcome (healthy or patient) and thermograms results (mean, median, mode, SD and skewness of the temperature of each breast). The output values were obtained by the following steps: the weight is adjusted for each processing element; the sum of all defined weights is calculated and used for one activation function that converts this value in an output value. This charge distribution process is known as normalized cumulative delta learning rule and was defined some default values ( $\mathrm{Ng}$ et al. 2002), which are a momentum of 0.4 and learning coefficients of hidden layer, output layer and ratio of $0.3,0.15$ and 0,5 respectively.

A different file was conceived to be applied in each ANN during the training phase. These four files had different basic variables. Next topics describes the content in each file, given by its inputs ( $\mathrm{Ng}$ et al. 2002):

- File 1: mean, median, modal, SD and skewness temperature of both breasts.

- File 2: mean, median and modal temperature of both breasts.

- File 3: age, family history, hormone replacement therapy, age of menarche, presence of palpable lump, previous surgery biopsy, presence of nipple discharge, breast pain, menopause at age $>50$ years, first child at age $<30$ years of the patient.

- File 4: the junction of files 2 and 3 .

The activation function is based in hyperbolic tangent and with this the results were in the range $[0,1]$ but then were normalized for to have the value 1 (means diseased patient) or -1 (healthy patient). To ensure a correct training process, it was calculated the Root Mean Square Error (RMSE) by the learning index. The hidden nodes number was defined in a trial error mode for each ANN, in other words, each ANN was tested with a different hidden nodes number for their basic variables file and then the RMSE was calculated ( $\mathrm{Ng}$ et al. 2002).

The results show that it is possible for an ANN to memorize and to generalize the relation between the system input and output data and the best results was obtained in the network that used the fourth basic variables file (File 4). This means that one advantage achieved with this work was the pattern recognition. Otherwise, some disadvantages were also found such as the lower precision results caused by the use of numeric values for the input data and the time spent in training process. However, after the conclusion of this process it only needed one cycle (epoch) for classifying or detecting a specific condition.

A total of 207 subjects were used, being 76 healthy, 98 benign and 33 malignant patients, the system has as highest sensitivity $68.97 \%$, specificity of $80 \%$ and accuracy of $61.94 \%$ ( $\mathrm{Ng}$ et al. 2002). 
Two years later, another publication (Jakubowska et al. 2004) with a similar method was developed having the same objectives and using ANN but, in this case, NDA, LDA and PCA statistical procedure techniques were used to reduce variables and compared.

The basic variables were defined with the following data captured (Jakubowska et al. 2004):

- Histograms of breast ROIs: mean, variance, skew ness, kurtosis and five histogram percentiles.

- Co-occurrence matrix based characteristics which are specified for matrices constructed for 5 distances between image pixel $(\mathrm{d}=1,2,3,45)$ and separately for the 4 directions: horizontal, vertical, 45 and 135 (angular second moment, contrast, correlation, sum of squares, inverse difference moment, sum average, sum variance, sum entropy, entropy, difference variance, difference entropy);

- Gradient-based elements (absolute gradient mean, variance, skewness, kurtosis and of non-zero gradients)

- Run-length matrix-based traits (short run emphasis inverse moment, long run moment, grey level no uniformity, run length no uniformity and fraction of in runs, for the same directions as in the case of co-occurrence matrix based features).

The mentioned histograms were captured from 30 healthy patients and 30 disease subjects. The results obtained with the application of data only from histogram were not conclusive. This happened because the implemented ANN had implicit in their architecture data reduction that caused a reduced multidimensional distance between the output results, this means that the probability of errors occurring at normalization is high and classification could be wrong (Jakubowska et al. 2004).

At the same year, another study which configured an ANN with BP to classify breast thermograms. After the reduction of the subgroup defined from five variables to only two (mean temperature difference and standard deviation difference), the created ANN uses only one invisible node (hidden node) and the obtained result was two false positives. According to the authors, the system failure is due to the use of few images from users where the cancer problem exists and, thus, the recruitment of more participants was advised in order to obtain better results (Koay et al. 2004).

In 2008, the pioneer in introducing AI classifiers in MITI has tried to prove that $\mathrm{BC}$ early diagnostic was feasible using ANN and some bio-statistical methods such as linear regression and ROC (Ng and Kee 2008).

The method created suggest the usage of a linear regression multiple mod-el, radial basis function network (RBFN) and an analysis by ROC. The regression allows the correlation between the variables and the currently state of the patient and this enables the correct selection of the features that were used in the training epoch for the ANN. Then, the activation function RBFN was used for to determine the classification result. This function, comparatively to $\mathrm{BP}$, presented that 
the training epoch is faster. This approach presented rates of $80.95 \%, 100 \%$ and $70.6 \%$ for accuracy, sensitivity and specificity correspondingly in identifying BC, which was largely higher compared with those parameters obtained from clinical examination conducted by experienced radiologists, which had an accuracy rate between 60 and $70 \%$ ( $\mathrm{Ng}$ and Kee 2008).

At the same year, a study aiming to automatically identify - Carpal Tunnel Syndrome (CTS) has used a learning group, which was produced by computer through random selection and represents a total of $60 \%$ of the 34 hands. Before starting the workout, the temperature of each individual portion was standardized based on the average temperature of the whole pertaining hand. This assured that compelling differences in the temperatures of hands between distinct people did not cause an influence on the classification outcome (those temperature differences within individuals are common and usually not related to any pathologic state). The leftover cases were then object of classification (Papež et al. 2008).

Different sorting attempts were performed, where the number of hand segments was changed, and these were used as input values in ANN. For each time, it was used a multilayer perceptron fully connected with two hidden layers (with nine neurons in the first hidden layer and five neurons in the subsequent hidden layer), followed by 100 training repetitions (where each hand of the training set was used 100 times). Each data classification was repeated five times, each being a training set randomly selected from $60 \%$ of the 34 hands, with the percentage of successful classifications being calculated based on the average of these five trials. In order to reduce the errors of comparison, a reference classification was used, where all 12 segments of the dorsal hand were included (Papež et al. 2008).

For a reference classification, the 12-existing hand dorsal segments were used, with an average success rate of $80.6 \%$ (18 out of 22 hands). Palm segments did not have any beneficial influence on the result of the classification, since the success rate increased considerably when they were omitted, while on the other hand when the dorsal segments were omitted, the opposite occurred. This led to an attention to the dorsal side over the palmar side of the hand. The segments of the fingers appeared to be of greater importance, especially the second (indexer) and the fifth (small) finger. When the area corresponding to the median nerve influence was suppressed (the first, second and third fingers), the success rate of the classification was similar to that of the random method. Thus, the metacarpal segments seem to bring benefits, since the classification worsens slightly when they are omitted. Controversially, the segments of the pulse when not considered, result in an improvement in the success rate, which leads to infer that these are not important for the correct classification based on the thermal scale (Papež et al. 2008).

In the following year, the same authors added a total of 502 images of hands to train the ANN and repeated the experience, they have found that based on the dorsal images of the hands, it was possible to correctly classify a total of $72.2 \%$ of all hands (healthy and pathological), being this about $80 \%$ when only healthy and seriously affected hands were included. Comparing these results with those obtained 
by the gold standard electromyographic diagnosis of CTS, the MITI technique cannot be recommended as an adequate diagnostic tool when accurate determination of severity level is required. However, the authors suggest that it could be used as a screening tool for identifying severe cases in populations with high ergonomic CTS risk factors (Papež et al. 2009).

In 2015, a study (Koprowski, 2015) proposed a method of image analysis for the evaluation of the temperature distribution along the back of the back-pain patients in which the images were collected. The main objective would be to detect anomalies that cause a defective posture. Some classifiers were used to classify the collected parameters, among which ANN. The results obtained show that for ANN with the use of BP, with 16 neurons in the invisible layer and learning by cross-validation, a sensitivity and specificity of $75 \%$ and $89 \%$ respectively was obtained. However, this was not the classifier with the highest values in the evaluation. The classifier with the highest success percentage value was the SVM with $88 \%$ and $90 \%$ respectively.

In the same year, another study (Koukiou and Anastassopoulos 2015) related the application of ANN in infrared images in order to identify people in a state of drunkenness. In order to reach the proposed aim, two approaches were adopted. In the first, using the ANN, the temperatures by location in each one of the different images from the same face were examined for all the collected faces, with this, it was verified that it was in the frontal images of the face where there was a major change in the thermal behavior with alcohol consumption. In the second, a singlestructure neural network was constructed with the basic building variables from all face locations, thus a highly discriminative performance was observed when applied to unknown data. However, smaller ANN presented better overall performance, with an overall accuracy of $86 \%$.

In 2016, Saniei et al. (Saniei et al. 2016) presented a new approach to predict an estimation of the size, depth and rate of metabolic heat generation in a tumor. This new approach is composed of two stages: progressive and inverse. For the first step, it was necessary to create a finite element model and solve the equation (The Pennes bio-heat) to calculate the surface and depth temperature distribution. The calculated values obtained were applied for the construction of a dynamic neural network (DNN) and, in order to ensure the correct network construction, the results of this application confirm the advantages of the finite element model. In the second action, the constructed network was used to estimate the temperature distribution in depth from the surface temperature profile

In order to avoid that the created network was too tight and in order to obtain a system with an acceptable level of performance, the authors considered as crucial the choice of the amount of neurons in the invisible layer and of the necessary times, values, were not dependent on each other but determined iteratively. There was a strong indication of the linear relationship between two variables was found to be 0.84 and 0.71 , respectively, for size and depth. Ab-solute errors in depth and size were within $0.31 \mathrm{~cm}$ and $0.2 \mathrm{~cm}$, respectively. Results showed that the depth estimation error was larger than the size estimation error, which influenced that 
deep seated tumors had higher error than other cases. More images are required for better assessment of the developed system (Saniei et al. 2016).

\subsection{SVM}

The second most experimented classifier of data in MITI was the SVM, which has the concept based on statistical learning theory and was applied to a set of classification methods that analyze data and recognize patterns. This automatic constructive learning procedure is based on the principle of structural risk minimization, that is, it intends to minimize existing errors on the data that are not included in the construction algorithm. Its advantage is due to the limits (borderlines) created by the automatic learning machine during the construction phase (Abe 2005, Wang 2005).

In general, an SVM classifier produces binary linear results, it analyses a set of initial data and assignments to one of two possible categories. Normally, this algorithm works well when applied to new data because the data applied during the build phase are generally the most difficult to classify. It implies that methods that choose to use this technique have the ability to overcome competing methods in many applications. In a representation model, the SVM uses a point mapping in space, and there is, in the created representation space, a hyperplane that separates the two classes. In this way, the points are distributed in the space according to the class belonging (Fig. 5) (Abe 2005, Wang 2005).

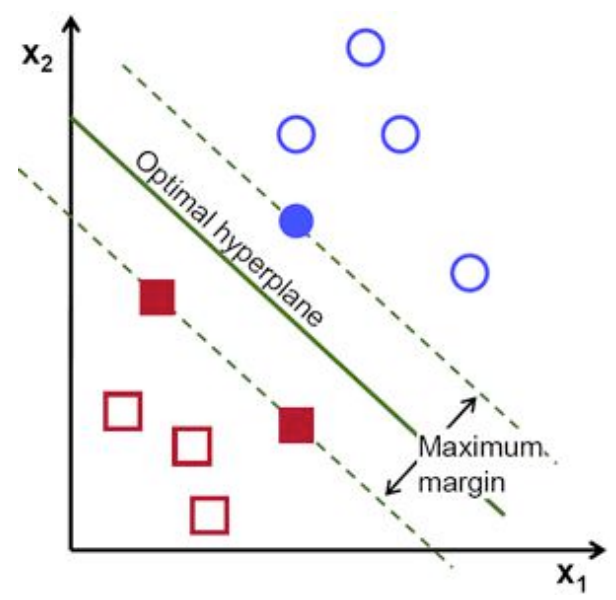

Fig. 5 Illustrative view of an example of a SVM.

The work of Archarya et al. (Archarya et al. 2012) aimed to evaluate the viability of use classified thermography in BC diagnostic. Texture characteristics were obtained from co-occurrence and run length matrices. After normalization process, the next step was to classify with application of SVM. The choice of this classifier 
was justified with the good performance obtained at previous studies in pattern recognition.

In the implementation of SVM, the Tree-fold stratified cross validation method was adopted, being the data divided in three sets. Then, for each combination of two sets, the SVM was trained and tested in the third set that remained in each combination. A result of $88.10 \%, 85.71 \%$ and $90.48 \%$ was obtained for accuracy, sensitivity and specificity respectively (Archarya et al. 2012).

At the same year, Resmini et al. (Resmini et al.2012) researched the features in breast region thermal imaging that are in need for the classification and detection of BC. This complementary work had the purpose of improve the results from previous work with the addition of classifiers, techniques of selection and data information in the databases. First, for features research, it was crucial to extract statistic features, fractal dimension and geo-statistic features. In the approach adopted for this work it was necessary to reduce the features. For accomplish this requirement it was used two data reduction techniques: PCA and IGR. Classifiers were used for label the data from patients as being with or without the pathology presence.

There were defined three databases (DB), each one with different features and used for the classifiers tests. The "Old" DB contained the data used in previous work of the same author. The "New" DB has the same information of "old" plus new captured images. In this developed work was also investigated if the results are improved when the data contains only data of the features subtraction between left and right breasts of each patient. This information was saved in a third database called "difference" (Resmini et al. 2012).

For the classification tests, it was used the WEKA tool and, in the case of SVM, it was defined the following parameters (Resmini et al. 2012):

- SVMType = nu-SVC;

- $\mathrm{nu}=0,5$;

- kernelType = linear;

For the remaining parameters, there were used the default settings of WEKA. For the evaluation of the results was considered two measurements: percent value of accuracy and area under the ROC curve (equal to sensibility/(1-specificity)). The obtained results showed an accuracy of $82.35 \%$ in the "New" DB (Resmini et al. 2012).

A research aiming to classify the Dry Eye disease with SVM collected thermal images in people with the problem and people without this pathology. More specifically, from the thermograms, statistical data such as energy, kurtosis, skewness, mean, standard deviation and various values of entropy were collected (Archarya et al. 2014).

With the SVM algorithms used the initial data collected from the thermo-grams were mapped in a dimensional space. Kernel functions in the order of 1 to 3 and RBF were used. The better results presented with SVM were with the polynomial 
function of order 3 , which had an accuracy of $84.76 \%$. This result was overcome by the k-NN classifier (Archarya et al. 2014).

Milosevic et al. (Milosevic et al. 2014) aimed to classify breast thermogram through applying SVM classifiers in the images for the detection of abnormal mass in tissue. In the application of the algorithm, a diagonal covariance (five-fold cross validation) matrix was adopted to ensure that the construction was performed exhaustively with all applied data.

As a procedure, the data were randomly divided by $k=5$ sets. The construction is performed with each set (k-1) and the result is applied to the remaining assemblies in test form. After this method, has been applied to all construction data sets and the consequent $\mathrm{K}$ tests were performed, an average of all $\mathrm{K}$ results is calculated, which means the final result of the classification. The SVM had the second highest score in this application, an accuracy of $85 \%$ only surpassed by the k-NN, which obtained a value of $92.5 \%$ (Milosevic et al. 2014).

In 2015, Acharya et al. (Acharya et al. 2015) revisited their research in Dry eye disease classification, improving the methodology, reducing the features for classification. The SVM presented an average sensitivity, specificity and classification accuracy of $99.9 \%, 99.4 \%$ and $99.8 \%$ respectively using SVM classifier with polynomial order 2 kernel, whether the k-NN classifier obtained the average sensitivity, specificity and classification accuracy of $99.8 \%$.

Sathish et al. (Sathish et al. 2016) presented a new approach to the analysis of breast thermograms, which consists in the development of fully automated segmentation, in order to be able to carry out an asymmetric analysis with respect to the respective side of the thermogram. The results obtained show a $90 \%$ accuracy, $87.5 \%$ sensitivity and $92.5 \%$ specificity were obtained for the 80 images applied to SVM.

\section{3 k-NN}

The k-NN algorithm can be used either for classification and/or clustering, it is classified as a supervised learning algorithm (Larose 2005).

As previously mentioned in the previous section in the dry eye disease (Archarya et al. 2014, Archarya et al. 2015) and breast classification (Milosevic et al. 2014) studies, the k-NN classifier obtained better efficacy results than the SVM.

In 2016, a study (Bhowmik 2016) on classification of thermograms with Osteoarthritis (OA), which is the most common manifestation of arthritis, and rheumatoid arthritis (RA) in the knee in 15 patients with $\mathrm{k}-\mathrm{NN}$ has been published. The authors followed a strict images capture protocol, after a few parameters (first and second order statistics) were extracted from the ROIs (affected and non-affected) such as mean temperature, standard deviation, entropy, kurtosis, skewness and variance (first order statistics); and homogeneity, contrast, energy, correlation, entropy and maximum probability (second order statistics). It has to be noted that inflammation occur in unilateral knee in cases of OA and in bilateral knee when RA 
exists. Taking into account the differences in the propagation of inflammation, the mean standard deviation and the contrast value of the intensity distribution on the IR images, these are able to discriminate between the affected knee and the healthy knee in the OA classification. In the categorization of RA, bearing in mind that the spread of inflammation in the bilateral joints is very similar, then the difference in statistical characteristics is smaller between the contralateral knees, making its identification more difficult.

Another recent research (Glowacz and Glowacz 2016) on applying k-NN classifiers to identify finger skin injuries using signal processing techniques such as extraction of magenta color, calculation of histogram, image filtration and calculation of perimeter. A total of 15 images of finger were used to train the classifier and 60 images of finger were used to test the proposed system. The best results were obtained for $\mathrm{k}=1$ and $\mathrm{k}=3$ and image filtration, histogram and $\mathrm{k}-\mathrm{NN}(100 \%)$.

\subsection{Fuzzy Logic}

The Fuzzy Logic is based on a human like interpretation for solving situations and thus the programming language used is very easy and simple to understand. Fuzzy logic incorporates a simple, rule-based IF X AND Y THEN Z approach in solving problems rather than performing models of solutions. The main issue of the fuzzy logic is its dimensionality, if the number of rules increases exponentially with respect to the number of attributes involved, it can become highly computational and memory expensive (Barro and Marín 2013).

A study (EtehadTavakol et al. 2010) used a 2-color segmentation approach, Kmeans and fuzzy c-means (FCM) for color segmentation of infrared breast images were modelled and compared. FCM were preferred because the fuzzy nature of IR breast images helps the FCM segmentation to provide more accurate outcomes with no empty cluster. A total of 6 thermal cases of BC were investigated, through using FCM, it was possible to find the 1st and the 2nd hottest regions for each case where some useful characteristics could be extracted from. There are several advantages in the color segmentation of thermal infrared breast images using FCM, such as: identifying abnormal cases by comparing the corresponding groups to the left and right sinuses, determining the degree of malignancy with the discovery of the two hottest regions and extracting parameters of these. The authors suggested to analyze the probability of development of the CB based on the characteristics used in the methodology developed by them (EtehadTavakol et al. 2010).

In the following year, a study (Sebiskveradze et al. 2011) aiming to develop a new FT-IR spectral imaging system of tumor tissue that allows a better characterization of the heterogeneity between the tumor and its surrounding tissue. Infrared data were acquired from 13 biopsies of paraffin embedded human skin carcinomas. The methodology used was based on an innovative FCM-based clustering algorithm, allowing automatic and simultaneous estimation of optimal FCM param- 
eters (number of clusters $\mathrm{K}$ and diffusion index $\mathrm{m}$ ). With the methodology proposed and developed, it was possible to emphasize a marked heterogeneity in the tumor and to characterize the interconnection between the structures of the tissue.

The unique known application (Schaefer et al. 2009) of Genetic Algorithms in medical applications of MITI was in breast thermograms classification, where it was used to reduce the number of rules of fuzzy logic, presenting accuracy of $80.98 \%$, through decreasing by $250 \%$ of number of required rules, the gain in accuracy was of about $1 \%$.

\subsection{NB}

NB is a simple probabilistic classifier, which is based on the application of Bayes' theorem, with strong (naive) assumptions of independence between resources. It only requires a small amount of training data to be able to estimate the parameters needed to classify new data (Murphy 2006).

There are three known applications of NB classifier in MITI application in breasts cancer classification, although in all of them the accuracy was low, in a study (Nicardo et al. 2013) it was of $71.86 \%$ and in the other two studies (Archarya et al. 2014, Archarya et al. 2015) this classifier had 3\% (Archarya et al. 2014) and $11 \%$ (Archarya et al. 2015) less accuracy when compared with SVM after reducing the variables.

\subsection{Adaboost}

Adaptive Boosting is a machine-learning meta-algorithm that can be used as a complement to other types of learning algorithms in order to improve performance. The outputs of the other learning algorithms ("weak learners") are then combined into a weighted sum representing the final output of the reinforced classifier. Thus, AdaBoost translates into an adaptive approach in the sense that subsequent weak learners are modified in favor of instances misclassified by previous classifiers. This classifier is highly sensitive to data and noisy outliers. However, it may also be less susceptible to the overlapping problem than other classifiers. (Freund, 1999).

The unique known application (EtehadTavakol et al. 2013) to date of Adaboost classification in MITI was published in 2013 and aimed to classify breast thermograms, a data set composed of 9 malignant, 12 benign and 11 normal cases of breast thermal images was used for evaluation of performance. The malignant cases presented a $95 \%$ accuracy rate after advanced features extraction, such as normalized bispectrum, which discards all magnitude information, demonstrating a better discrimination between benign and normal cases, with $83 \%$ of accuracy rate when compared to $66 \%$ obtained with the original. 


\section{Discussion}

From the presented literature, it can be easily identified that most of the application of artificial intelligence (AI) classifiers in MITI is in BC identification (table 2), all of the above outlined classifiers have been tested in that application, the one that showed better results was the k-NN, followed closely by Adaboost and SVM with accepted accuracy rates. Nonetheless, it worth's to mention that despite of six decades of research in the fields of MITI in BC identification, to date no convincing standard clinical application was successful, this is due that at an early stage any abnormality at skin surface temperature can be related with any other cause than BC. From the table 2 it can be observed that the areas of the human body that had more interest in being studied were the breast, head, hands, knee, fingers and back. The major advantages of the studied classifiers were the pattern recognition and the features reduction and the limitations were the low precision and small samples.

Table 2. Comparison between classifiers and their application in MITI.

\begin{tabular}{|c|c|c|c|c|c|c|c|}
\hline Application & Classifier & Body part & Advantages & Disadvantages & Accuracy & Sensitivity & Specificity \\
\hline $\mathrm{BC}$ & ANN & Breast & $\begin{array}{l}\text { Pattern } \\
\text { recognition }\end{array}$ & Low precision & $61.94 \%$ & $68.97 \%$ & $80 \%$ \\
\hline $\mathrm{BC}$ & ANN & Breast & & $\begin{array}{l}\text { Not conclusive } \\
\text { and great } \\
\text { probability of } \\
\text { errors }\end{array}$ & & & \\
\hline $\mathrm{BC}$ & ANN & Breast & & Few images & & & \\
\hline $\mathrm{BC}$ & ANN & Breast & $\begin{array}{l}\text { Correct se- } \\
\text { lection of } \\
\text { features }\end{array}$ & & $80.95 \%$ & $100 \%$ & $70.60 \%$ \\
\hline CST & ANN & Hands & & $\begin{array}{l}\text { Poor selection } \\
\text { of features and } \\
\text { small sample }\end{array}$ & $80.60 \%$ & & \\
\hline CST & ANN & Hands & $\begin{array}{l}\text { Good } \\
\text { screening } \\
\text { tool }\end{array}$ & $\begin{array}{l}\text { Poor diagnos- } \\
\text { tic method }\end{array}$ & $\begin{array}{l}72.20 \% \\
\text { and } 80 \% \\
\text { (severe } \\
\text { cases) }\end{array}$ & & \\
\hline Back pain & ANN & Back & & & & $75 \%$ & $89 \%$ \\
\hline Back pain & SVM & Back & & & & $88 \%$ & $90 \%$ \\
\hline $\begin{array}{l}\text { State of drunk- } \\
\text { ness }\end{array}$ & ANN & Head & & & $86 \%$ & & \\
\hline $\mathrm{BC}$ & SVM & Breast & $\begin{array}{l}\text { Pattern } \\
\text { recognition }\end{array}$ & & $88.10 \%$ & $85.71 \%$ & $90.48 \%$ \\
\hline $\mathrm{BC}$ & SVM & Breast & $\begin{array}{l}\text { Reduction of } \\
\text { features }\end{array}$ & & $82.35 \%$ & & \\
\hline $\mathrm{BC}$ & NB & Breast & Reduction of & & $79.35 \%$ & & \\
\hline
\end{tabular}




\begin{tabular}{|c|c|c|c|c|c|c|}
\hline & & & features & & & \\
\hline Dry eye disease & SVM & Head & & $84.76 \%$ & $86.14 \%$ & $83.33 \%$ \\
\hline Dry eye disease & $\mathrm{k}-\mathrm{NN}$ & Head & & $99.88 \%$ & $99.76 \%$ & $100 \%$ \\
\hline Dry eye disease & NB & Head & & $65.73 \%$ & $77.23 \%$ & $53.95 \%$ \\
\hline $\mathrm{BC}$ & SVM & Breast & & $85 \%$ & & \\
\hline $\mathrm{BC}$ & $\mathrm{k}-\mathrm{NN}$ & Breast & & $92.5 \%$ & & \\
\hline $\mathrm{BC}$ & NB & Breast & & $81.5 \%$ & & \\
\hline Dry eye disease & SVM & Head & & $99.8 \%$ & $99.9 \%$ & $99.4 \%$ \\
\hline Dry eye disease & $\mathrm{k}-\mathrm{NN}$ & Head & & $99.8 \%$ & $99.8 \%$ & $99.8 \%$ \\
\hline Dry eye disease & NB & Head & & $89.46 \%$ & $92 \%$ & $79.39 \%$ \\
\hline $\mathrm{BC}$ & SVM & Breast & $\begin{array}{l}\text { Fully auto- } \\
\text { mated seg- } \\
\text { mentation }\end{array}$ & $90 \%$ & $87.5 \%$ & $92.5 \%$ \\
\hline $\begin{array}{l}\text { Osteoarthritis } \\
\text { and Rheumatoic } \\
\text { Arthritis }\end{array}$ & $\mathrm{d}^{\mathrm{k}-\mathrm{NN}}$ & Knee & $\begin{array}{l}\text { OA good RA poor per- } \\
\text { performance formance }\end{array}$ & & & \\
\hline $\begin{array}{l}\text { Finger skin in- } \\
\text { jury }\end{array}$ & k-NN & finger & $\begin{array}{l}\text { Feature se- } \\
\text { lection }\end{array}$ & $100 \%$ & & \\
\hline $\mathrm{BC}$ & $\begin{array}{l}\text { Fuzzy log- } \\
\text { ic }\end{array}$ & -Breast & Sample size & & & \\
\hline $\mathrm{BC}$ & $\begin{array}{l}\text { Fuzzy log- } \\
\text { ic }\end{array}$ & -Breast & $\begin{array}{l}\text { Identified } \\
\text { the marked } \\
\text { heterogenei- } \\
\text { ty }\end{array}$ & & & \\
\hline $\mathrm{BC}$ & $\begin{array}{l}\text { Fuzzy log- } \\
\text { ic }\end{array}$ & -Breast & $\begin{array}{l}\text { GA reduced } \\
\text { the varia- } \\
\text { bles, and } \\
\text { rules by } \\
250 \%\end{array}$ & $80.98 \%$ & & \\
\hline $\mathrm{BC}$ & NB & Breast & & $71.86 \%$ & & \\
\hline \multirow[t]{2}{*}{$\mathrm{BC}$} & Adaboost & Breast & & $\begin{array}{l}\text { Malignant } \\
95 \%\end{array}$ & & \\
\hline & & & & $\begin{array}{l}\text { Distinc- } \\
\text { tion } 83 \%\end{array}$ & & \\
\hline
\end{tabular}

A good example of application of intelligent data classifiers in MITI is the classification of OA and RA (Bhowmik et al. 2016), which has shown an accuracy of $100 \%$ despite the small size of the sample to be classified. Other good example of application of AI classifiers in MITI was the identification of Dry Eye disease (Archarya et al. 2014, Archarya et al. 2015) either with k-NN and SVM.

The classification of back pain (Koprowski 2015) and drunkenness (Koukiou and Anastassopoulos 2015) states in infrared images with intelligent classifiers presented an accepted accuracy, however if the sample images increase those values may improve significantly. 
The application of ANN in the classification of CTS in thermal images (Papež et al. 2008, Papež et al. 2009) had a significant sample size, although the authors considered that their approach was not recommended for diagnosis but it was worthily for monitoring.

The use of Fuzzy logic classifiers demonstrated to be helpful for features extraction.

The inexistence of healthy reference data and standard ROIs in MITI is the major barrier for the successful application of AI classifiers.

There is no doubt about the utility and usefulness of data classifiers in medical imaging applications (Dey 2016). The application of intelligent data classifiers in MITI is still unexplored, due to some barriers such as the unfamiliarity of the imaging technique by the health professionals and its principles and the lack of a standard imaging file format, which difficult data exchange and integration into information systems and development of advance CAD tools.

Examples of MITI applications that could have a great success in using intelligent data classifiers are: diabetic foot ulcers, Raynaud's phenomenon, soft tissues rheumatism, blood pressure, Hand-Arm Vibration Syndrome, peripheral nerves compressions, Complex Regional Pain Syndrome, fever screening, burns (tissue viability), Dermatological disorders, temporomandibular joint conditions, thyroid dysfunctions, renal dialysis, skin cancer, chemotherapy assessment and rehabilitation medicine procedures assessment.

\section{Acknowledgements}

The authors thank the partial funding of Project NORTE-01-0145-FEDER000022 - SciTech - Science and Technology for Competitive and Sustainable Industries, co-financed by Programa Operacional Regional do Norte (NORTE2020), through Fundo Europeu de Desenvolvimento Regional (FEDER) and the FCT Foundation for Science and Technology under the project (PEstOE/EME/LA0022/2013).

\section{References}

Abe S (2005) Support vector machines for pattern classification. Volume 2. London: Springer. Acharya UR, Ng EYK, Tan JH, Sree SV(2012) Thermography based breast cancer detection using texture features and support vector machine. Journal of medical systems 36(3): 15031510

Acharya UR, Tan JH, Vidya S, Yeo S, Too CL, Lim WJE, Chua KC, Tong L (2014) Diagnosis of response and non-response to dry eye treatment using infrared thermography images. Infrared Physics \& Technology, 67: 497-503. 
Acharya UR, Tan JH, Koh JE, Sudarshan VK, Yeo S., Too CL, Chua CK, Ng EYK, Tong L (2015) Automated diagnosis of dry eye using infrared thermography images. Infrared Physics \& Technology, 71:263-271.

Ammer K (2003) Need for standardisation of measurements in thermal imaging. Thermography and Lasers in Medicine. Akademickie Centrum Graficzno-Marketigowe Lodart SA, Lodz (Poland), 13-18

Ammer K (2008) The Glamorgan Protocol for recording and evaluation of thermal images of the human body. Thermology international 18(4): 125-144

Bezdek JC, Ehrlich R, Full W (1984) FCM: The fuzzy c-means clustering algorithm. Computers \& Geosciences 10(2-3): 191-203.

Barro S, Marín R. (Eds.) (2013) Fuzzy logic in medicine. Volume 83. Physica.

Bhowmik MK, Bardhan S, Das K, Bhattacharjee D, Nath S (2016) Pain related inflammation analysis using infrared images. In SPIE Commercial+ Scientific Sensing and Imaging. International Society for Optics and Photonics. 986116-986116

Dey N (Ed.) (2016) Classification and clustering in biomedical signal processing. IGI Global

EtehadTavakol M, Sadri S, Ng EYK (2010) Application of K-and fuzzy c-means for color segmentation of thermal infrared breast images. Journal of medical systems 34(1): 35-42

EtehadTavakol M, Chandran V, Ng EYK, Kafieh R (2013) Breast cancer detection from thermal images using bispectral invariant features. International Journal of Thermal Sciences 69: 2136

Freund Y, Schapire R, Abe N (1999) A short introduction to boosting. Journal-Japanese Society for Artificial Intelligence 14(771-780): 1612

Glowacz A, Glowacz Z (2016) Recognition of images of finger skin with application of histogram, image filtration and K-NN classifier. Biocybernetics and Biomedical Engineering 36(1): $95-101$

Hassoun MH (1995) Fundamentals of artificial neural networks. MIT press

Howell KJ, Smith RE (2009) Guidelines for specifying and testing a thermal camera for medical applications. Thermology international 19(1): 5-14

ISO, TR13154 (2009) Medical Electrical Equipment - Deployment, implementation and operational guidelines for identifying febrile humans using a screening thermograph.

Jakubowska T, Wiecek B, Wysocki M, Drews-Peszynski C, Strzelecki M (2004) Classification of breast thermal images using artificial neural networks. In Engineering in Medicine and Biology Society, 2004. IEMBS'04. 26th Annual International Conference of the IEEE, 11551158.

Jones BF, Plassmann P (2002) Digital infrared thermal imaging of human skin. IEEE Engineering in Medicine and Biology Magazine 21(6): 41-48

Koay J, Herry C, Frize, M (2004) Analysis of breast thermography with an artificial neural network. In Engineering in Medicine and Biology Society, 2004. IEMBS'04. 26th Annual International Conference of the IEEE, 1159-1162

Koprowski R (2015) Automatic analysis of the trunk thermal images from healthy subjects and patients with faulty posture. Computers in biology and medicine 62: 110-118

Koukiou G, Anastassopoulos V (2015) Neural networks for identifying drunk persons using thermal infrared imagery. Forensic science international 252: 69-76

Larose DT (2005) k - Nearest Neighbor Algorithm. Discovering Knowledge in Data: An Introduction to Data Mining, 90-106.

Lawson R (1956) Implications of surface temperatures in the diagnosis of breast cancer. Canadian Medical Association Journal 75(4): 309-310

Lisowska-Lis A, Mitkowski SA., Augustyn J (2011) Infrared technique and its application in science and engineering in the study plans of students in electrical engineering and electronics. In Proceedings of 2 nd world conference on technology and engineering education (WIETE 2011): 104-108

Lloyd JM (2013) Thermal imaging systems. Springer Science \& Business Media 
Milosevic M, Jankovic D, Peulic A (2014) Thermography based breast cancer detection using texture features and minimum variance quantization. EXCLI journal 13: 1204.

Murphy KP (2006) Naive bayes classifiers. University of British Columbia.

Ng EYK, Fok SC, Peh YC, Ng FC, Sim LSJ (2002) Computerized detection of breast cancer with artificial intelligence and thermograms. Journal of medical engineering \& technology 26(4): $152-157$

$\mathrm{Ng}$ EYK, Kee EC (2008) Advanced integrated technique in breast cancer thermography. Journal of medical engineering \& technology 32(2): 103-114

Nicandro CR, Efrén MM, María Yaneli AA, Enrique MDCM, Héctor Gabriel AM, Nancy PC, Alejandro GH, Guillermo de Jesús HR, Rocío Erandi BM (2013) Evaluation of the diagnostic power of thermography in breast cancer using bayesian network classifiers. Computational and mathematical methods in medicine, 2013.

Papež BJ, Palfy M, Turk Z (2008) Infrared thermography based on artificial intelligence for carpal tunnel syndrome diagnosis. Journal of International Medical Research 36(6): 1363-1370

Papež BJ, Palfy M, Mertik M, Turk Z (2009) Infrared thermography based on artificial intelligence as a screening method for carpal tunnel syndrome diagnosis. Journal of International Medical Research 37(3): 779-790

Qi H, Diakides NA (2007) Infrared imaging in Medicine

Resmini R, Borchartt TB, Conci A, Lima RC (2012) Auxílio ao Diagnóstico Precoce de Patologias da Mama Usando Imagens Térmicas e Técnicas de Mineração de Dados. Anais do Computer on the Beach, 305-314

Richards PL (1994) Bolometers for infrared and millimeter waves. Journal of Applied Physics 76(1): $1-24$

Ring EFJ, Ammer K (2000) The technique of infrared imaging in medicine. Thermology international 10(1): 7-14

Ring EFJ, Ammer K, Jung A, Murawski P, Wiecek B, Zuber J, Zwolenik S, Plassmann P, Jones $\mathrm{CD}$, Jones BF (2004) Standardization of infrared imaging. In Engineering in Medicine and Biology Society, 2004. IEMBS'04. 26th Annual International Conference of the IEEE, $1: 1183-1185$

Ring EFJ, Ammer K, Wiecek B, Plassmann P, Jones CD, Jung A, Murawski P (2005) Technical challenges for the construction of a medical image database. In Optical Systems Design 2005 International Society for Optics and Photonics. 59640N-59640N.

Ring EFJ (2007) The historical development of temperature measurement in medicine. Infrared physics \& technology 49(3): 297-301

Ring EFJ, Ammer K, Wiecek B, Plassmann P, Jones CD, Jung A, Murawski P (2007) Quality assurance for thermal imaging systems in medicine. Thermology International 17(3): 103-106

Ring EFJ, Ammer K (2012) Infrared thermal imaging in medicine. Physiological measurement 33(3): R33-R49

Saniei, E., Setayeshi, S., Akbari, M. E., \& Navid, M. (2016). Parameter estimation of breast tumour using dynamic neural network from thermal pattern. Journal of Advanced Research 7(6): 1045-1055.

Sathish D, Kamath S, Prasad K, Kadavigere R, Martis RJ (2016) Asymmetry analysis of breast thermograms using automated segmentation and texture features. Signal, Image and Video Processing, 1-8.

Schaefer G, Závišek M, Nakashima T (2009) Thermography based breast cancer analysis using statistical features and fuzzy classification. Pattern Recognition 42(6): 1133-1137.

Schwartz RG, Elliott R, Goldberg GS (2006) Guidelines for neuromusculoskeletal thermography. Thermology international 16(1): 5-9

Sebiskveradze D, Vrabie V, Gobinet C, Durlach A, Bernard P, Ly E, Manfait M, Jeannesson P, Piot $O$ (2011) Automation of an algorithm based on fuzzy clustering for analyzing tumoral heterogeneity in human skin carcinoma tissue sections. Laboratory Investigation 91(5): 799811 
TC121, ISO, SC62D, SI (2008) Particular requirements for the basic safety and essential performance of screening thermographs for human febrile temperature screening.

Thomas RA (1999) The thermography monitoring handbook. Coxmoor Publishing

Wang L (Ed.) (2005) Support vector machines: theory and applications. Volume 177. Springer Science \& Business Media

Williams KL (1964) Infrared thermometry as a tool in medical research. Annals of the New York Academy of Sciences 121(1): 99-112 